\title{
Robot-assisted laparoscopic adenomyomectomy: A review of literature
}

\author{
Ayah Hijazi ${ }^{1}$, Youn Jee Chung ${ }^{2}$, Jae Yen Song ${ }^{2}$, Mee-Ran Kim² \\ ${ }^{1}$ Department of Obstetrics and Gynecology, King Abdulaziz Medical City, Ministry of National Guard-Health Affairs, Riyadh, Kingdom of Saudi Ara- \\ bia; '2Department of Obstetrics and Gynecology, Seoul St. Mary's Hospital, College of Medicine, The Catholic University of Korea, Seoul, Korea
}

\begin{abstract}
Adenomyosis is a benign gynecologic disease characterized by the presence of endometrial glands and stroma within the myometrium. The reported prevalence is $5-70 \%$ among symptomatic women. Patients can exhibit various symptoms, but most likely present with heavy menstrual bleeding and dysmenorrhea. Management generally begins with medical treatment, in those who are refractory, hysterectomy has been the standard of treatment. However, uterine conserving options should be available for women who wish to maintain fertility. Robot-assisted laparoscopic adenomyomectomy is a relatively new technique used in the management of adenomyosis. We conducted a literature review using PubMed, Google Scholar, and MEDLINE (2000-2021) to define the role of robot-assisted laparoscopic adenomyomectomy in the conservative management of adenomyosis. The objective of this review is to summarize the role of robot-assisted laparoscopic adenomyomectomy and its current state. Robot-assisted adenomyomectomy may be an option that provides the benefits of a minimally invasive approach while allowing the surgeon to properly perform a technically challenging surgery. Data is still sparse and is of low quality, further prospective multicenter trials using a standardized technique and addressing the long-term surgical outcomes, effects on fertility and pregnancy outcomes will help further define the role of robot-assisted adenomyomectomy in the management of adenomyosis.
\end{abstract}

Key Words: Adenomyoma; Adenomyosis; Robotic surgical procedures

\section{INTRODUCTION}

Classified into diffuse and focal [1], adenomyosis is defined as the presence of endometrial glands and stroma within the myometrium [2-4]. These are surrounded by a reactive hyperplasia of the myometrial smooth muscle cells $[3,4]$. The prevalence of this disease varies widely and ranges between $5 \%$ to $70 \%$ in symptomatic women $[5,6]$. This disparity is due to the absence of standardized diagnostic criteria for imaging and pathologic testing [2].

Approximately two-thirds of patients with adenomyosis suffer from abnormal uterine bleeding and dysmenorrhea. Other symptoms include dyspareunia, acyclic pelvic pain, cyclic dysuria and dyschezia [7]. Adenomyosis also seems to have a negative impact on fertility [8], particularly in patients undergoing in vitro fertilization (IVF) treatments [3,8].

- Received: March 19, 2021 • Accepted: March 23, 2021

- Correspondening author: Mee-Ran Kim

Department of Obstetrics and Gynecology, Seoul St. Mary's Hospital, College of Medicine, The Catholic University of Korea, 222 Banpo-daero, Seocho-Gu, Seoul 06591, Korea

E-mail:drmrkim@gmail.com

This is an Open Access article distributed under the terms of the Creative Commons Attribution Non-Commercial License (http://creativecommons.org/ licenses/by-nc/4.0) which permits unrestricted non-commercial use, distribution, and reproduction in any medium, provided the original work is properly cited. 
Patients with adenomyosis undergoing IVF treatments have been found to have a reduced implantation and pregnancy rates, increased early pregnancy loss and, consequently, a lower live birth rate [3].

Diagnosing patients with adenomyosis presents a clinical challenge, not only do patients exhibit nonspecific symptoms, but also this disease is often associated with other pelvic pathologies such as leiomyomas [9], which co-exist in about $40 \%$ of patients [10]. Bazot et al. [9] found that transabdominal ultrasound was less accurate than transvaginal ultrasound for the diagnosis of adenomyosis when correlated with histological findings. In women without other pelvic disorders, transvaginal ultrasound has a similar accuracy in diagnosing the disease as does magnetic resonance imaging (MRI). However, transvaginal ultrasonography becomes less accurate in women with associated leiomyomas [9].

Management of adenomyosis in reproductive aged women usually involves the use of medical treatment including prostaglandin inhibitors, progestogens, combined oral contraceptives, and gonadotropin-agonists [11]. Although medical management can cause a transient regression of disease, women seeking fertility may better be treated surgically [5].

In a systematic review and meta-analysis, all studies showed an improvement in symptoms following conservative surgery. Pain and bleeding were both improved by more than $70 \%$ at 12 months. Complete excision of adenomyosis resulted in better improvement of pain, menorrhagia, and reduction of uterine volume, yet even after partial excision the improvements were substantial [12].

In a prospective study comparing surgery alone versus surgery followed by medical therapy, conservative surgery regardless of medical therapy relieved the severity of adenomyoma symptoms. With more than $90 \%$ of their patients satisfied with treatment by the end of the second year. However, the relapse rate was 20\% lower in the group that was also treated with a 6-month course of gonadotropinagonists postoperatively [13].

Since the year 2000 with the first report of robot use in gynecology [14], robot-assisted surgeries have come a long way in gynecology. From the surgical console, the surgeon is able to operate via remote-controlled arms. Gynecologic surgeries conducted with the aid of robot include benign surgeries such as hysterectomy, myomectomy, tubal anas- tomosis, sacrocolpopexy, endometriosis surgery, in addition to surgeries for gynecologic cancer, particularly endometria and cervical cancer [15].

The robotic system has several advantages over laparoscopy namely its 3-dimensional view, camera stability, tremor control, the ability to overcome the limitations of surgery for complex surgeries, and shorter learning curves. Surgical proficiency for robotic surgery was found to plateau at a lower number than for laparoscopic surgery (24 vs. 49) [16]. All this increases the use of a minimally invasive approach [17]. On the other hand, cost still remains a concern, in addition to the absence of tactile feedback, a drawback shared with the laparoscopic technique [17].

There is, however, limited data on the use of robot-assisted surgeries in adenomyomectomy. In particular, the best suture method, that will allow standardization of care for patients and enable safer reproductive outcomes. There is also little data on the reproductive outcomes of patients who have undergone robot-assisted laparoscopic adenomyomectomy.

\section{ROBOT-ASSISTED LAPAROSCOPIC ADENOMYOMECTOMY}

\section{Indications}

Indications for adenomyomectomy vary and include dysmenorrhea and heavy menstrual bleeding refractory to medical treatment in women seeking to preserve their uterus. Infertility and recurrent miscarriage are among other causes [18].

Laparoscopic management of adenomyosis may be more suitable for focal adenomyosis due to the limited direction of motion and limited instrument use [18]. However, in our experience with the benefits of robot-assisted surgeries, diffuse adenomyosis can also be managed with a minimally invasive approach.

\section{Surgical techniques}

Due to the nature of the disease, excision of adenomyosis is always accompanied by removal of normal myometrial tissue. Surgeons need to find a balance between the benefit of removing more adenomyotic tissue versus the disadvantage of leaving a defective uterine wall [4]. The dead space created following adenomyomectomy should be carefully 
closed, and the fallopian tubes should be left patent to allow for spontaneous pregnancy [11].

Chung et al. [19] reported four cases of robot-assisted laparoscopic adenomyomectomy. The Da Vinci surgical system (Intuitive Surgical, Sunnyvale, CA, USA) was used in their surgeries. After diluted vasopressin was injected into the overlying serosa, a 'cold-cut' technique using monopolar scissors with minimal energy was used. The myometrial defect was repaired layer-by-layer in an interrupted manner with the use of 2-O Vicryl ${ }^{\circledR}$ suture (Ethicon, Inc., Somerville, NJ, USA). The serosa was sutured in a baseball fashion.

A levonorgestrel-releasing intrauterine system was inserted for all patients immediately after surgery. The mean operative time was 159.25 minutes, mean estimated blood loss was $117.5 \mathrm{~mL}$, and the mean hospital stay was 2.5 days. Patients reported an improvement in dysmenorrhea and pelvic pain on 1-2 weeks follow up. All patients underwent a pelvic MRI 6 months following surgery and their MRI showed no residual disease. Patients were followed for about 2 years and were without symptoms.

In a prospective observational study, patients undergoing laparoscopic or robotic adenomyomectomy with uterine artery ligation for symptomatic adenomyosis were observed [20]. About half of the patients received a postoperative 3-month course of GnRH agonists. Eight of the 33 patients underwent robot-assisted adenomyomectomy while the rest underwent laparoscopy.

Uterine artery was ligated prior to adenomyomectomy, following that, diluted vasopressin was injected into the myometrium. A monopolar hook Harmonic ${ }^{\circledR}$ scalpel (Ethicon Endo-Surgery, Cincinnati, OH, USA) was used to make a vertical incision until the endometrial cavity was exposed. Adenomyomectomy was then carried out on either side of the incision. The double-flap method was used to close the dead cavity. A 2-0 Vicryl suture was used to close endometrial cavity, then left serosal flap was sutured to the right muscular layer using an absorbable glyconate monofilament (Monosyn ${ }^{\circledR}$ 0; B-Brown, Tuttlingen, Germany). Lastly, the right serosal flap was sutured over the left using an interrupted technique.

All patients had an improvement in their pain score, menorrhagia, and hemoglobin level. Three patients had a recurrence in their dysmenorrhea, and one had recurrence in menorrhagia by the end of the first year. There was no dif- ference in outcome between patients who had surgery alone versus those who had medical treatment in addition to surgery. In their study the operation and suturing time were longer in the robotic group.

They noted no statistical significant difference in blood loss, hemoglobin change, length of hospital stay or complications between those who underwent laparoscopy and those who had robotic surgery.

In an attempt to use an energy source with less lateral spread, Barton and Gargiulo [21] employed the use of flexible $\mathrm{CO}_{2}$ laser in 13 women with symptomatic leiomyomas and/or adenomyosis. The Da Vinci surgical system was used in their surgeries. After inflating the abdomen and docking of the robotic arms, diluted vasopressin was injected into the myometrium. The flexible $\mathrm{CO}_{2}$ laser was inserted through a right lower quadrant assistant port. The power was set to $15 \mathrm{~W}$ and with a no-touch technique the laser was used to create an incision over the adenomyoma. This was followed by enucleation of the pathology using the laser energy combined with traction. Following enucleation the defect was closed in multiple layers with barbed sutures (O QuillTM self-retaining system; Angiotech Pharmaceuticals, Inc., Vancouver, Canada or V-locTM absorbable wound closure device; Covidien, Dublin 2, Ireland). Only two of their patients had adenomyosis. Their median surgical time was 169 minutes with a median intraoperative blood loss of $25 \mathrm{~mL}$. None of the patients had intra or postoperative complications, and none required blood transfusion or overnight hospitalization.

\section{Postoperative outcomes}

There is limited data on the surgical outcomes following robot-assisted laparoscopic adenomyomectomy. However, conservative surgery outcomes can likely be generalized to various surgical techniques, including robot-assisted surgery.

More than three fourths of women undergoing conservative surgery will exhibit symptom relief [11]. Pregnancy rate after adenomyomectomy has been reported to be between $17.5-72.7 \%$, although this fairly high rate may be due to assisted reproductive technology [18].

Abnormal placentation and miscarriage rates do not appear to increase after conservative surgery for adenomyosis. The majority of pregnancies continue to proceed to a vi- 
able term delivery. The elected method of delivery is cesarean section, although there are cases of uncomplicated vaginal births [12].

\section{Uterine rupture risk}

The uterine rupture risk following adenomyomectomy appears to be higher than with other types of uterine surgeries. The risk in a vaginal birth following cesarean section has been reported to be between $0.27-0.7 \%$ [22] and following laparoscopic myomectomy $0.26 \%$ [23]. On the other hand the risk following adenomyomectomy is more than $1 \%$ [24] and has been reported to be as high as 6\% [18].

Osada reported 24 cases of uterine rupture in the years following 1,990 out of 2,365 case of adenomyomectomy. Laparoscopic adenomyomectomy seems to have an increased risk of uterine rupture following adenomyomectomy in comparison to laparotomy due to the incomplete repair of muscle defect [18]. Whether this technical difficulty can be overcome by the use of robot-assisted laparoscopy is still to be determined by further research.

At the time of this review, no cases of uterine rupture have been reported in pregnancies following robot-assisted laparoscopic adenomyomectomy. Having said that, robotassisted laparoscopic adenomyomectomy is still relatively new and the safety of this procedure cannot be confirmed without further research.

\section{Comparison with Laparoscopy}

Compared to robotic instruments, laparoscopic instruments offer a limited range of motion, an inadequate ergonomic handle grip, and an ineffective handle-tip force transfer. Robots can produce fast, accurate, and repetitive movements for extended operations while eliminating tremor and reducing surgeon fatigue [25].

In adenomyomectomy the lower degree of freedom makes laparoscopy more suitable for small localized adenomyosis. Larger myometrial defects may be difficult to repair properly and will need highly skilled surgeons.

In a retrospective study comparing robot-assisted adenomyomectomy to laparoscopic adenomyomectomy, no differences were observed in surgical time, blood loss, specimen weight and duration of hospital stay [6]. There were no post-operative complications in both groups. Although the surgeons were on their learning curve for robotic surgery, they concluded that there was no demonstrable benefit of robot-assisted surgery compared to laparoscopic adenomyomectomy in terms of surgical results.

Chong et al. [20] found that operative and suturing time was longer in robot-assisted adenomyomectomy compared to the laparoscopic approach. However, both groups had similar duration of hospital stay and intraoperative blood loss.

\section{CONCLUSION}

A conservative surgical approach for adenomyomectomy may be the therapy of choice for women of childbearing age. However, a standardized operative approach has yet to be described. Minimally invasive surgery has been proven to be associated with less intra and postoperative complications. Robot-assisted adenomyomectomy may provide a minimally invasive approach to a wider set of patients, because of the shorter learning curve and the ability to overcome the technical difficulty needed for meticulous suturing of the dead space. Yet this technique is still new, and data is of low quality. Randomized trials are still lacking and longer follow up is needed. Further prospective multicenter trials using a standardized technique and addressing the long-term surgical outcomes, effects on fertility and pregnancy outcomes are warranted.

\section{Conflict of interest}

No potential conflict of interest relevant to this article was reported.

\section{References}

1. Donnez J, Donnez O, Dolmans MM. Introduction: uterine adenomyosis, another enigmatic disease of our time. Fertil Steril 2018;109:369-70.

2. Struble J, Reid S, Bedaiwy MA. Adenomyosis: a clinical review of a challenging gynecologic condition. J Minim Invasive Gynecol 2016;23:164-85.

3. Younes G, Tulandi T. Effects of adenomyosis on in vitro fertilization treatment outcomes: a meta-analysis. Fertil Steril 2017;108:48390.e3.

4. Grimbizis GF, Mikos T, Tarlatzis B. Uterus-sparing operative treatment for adenomyosis. Fertil Steril 2014;101:472-87.

5. Garcia L, Isaacson K. Adenomyosis: review of the literature. J 
Minim Invasive Gynecol 2011;18:428-37.

6. Shim JI, Jo EH, Kim M, Kim MK, Kim ML, Yun BS, et al. A comparison of surgical outcomes between robot and laparoscopy-assisted adenomyomectomy. Medicine (Baltimore) 2019;98:e15466.

7. da Silva JR, Andres MP, Leite APK, Gomes MTNA, Neto JS, Baracat EC, et al. Comparison of sensitivity and specificity of structured and narrative reports of transvaginal ultrasonogaphy for adenomyosis. J Minim Invasive Gynecol 2020 Nov 15 [Epub]. https://doi.org/10.1016/j.jmig.2020.11.001.

8. Munro MG. Uterine polyps, adenomyosis, leiomyomas, and endometrial receptivity. Fertil Steril 2019;111:629-40.

9. Bazot M, Cortez A, Darai E, Rouger J, Chopier J, Antoine JM, et al. Ultrasonography compared with magnetic resonance imaging for the diagnosis of adenomyosis: correlation with histopathology. Hum Reprod 2001;16:2427-33.

10. Bazot M, Daraï E. Role of transvaginal sonography and magnetic resonance imaging in the diagnosis of uterine adenomyosis. Fertil Steril 2018;109:389-97.

11. Younes G, Tulandi T. Conservative surgery for adenomyosis and results: a systematic review. J Minim Invasive Gynecol 2018;25:265-76.

12. Mikos T, Lioupis M, Anthoulakis C, Grimbizis GF. The outcome of fertility-sparing and nonfertility-sparing surgery for the treatment of adenomyosis. A systematic review and meta-analysis. J Minim Invasive Gynecol 2020;27:309-31.e3.

13. Wang PH, Liu WM, Fuh JL, Cheng MH, Chao HT. Comparison of surgery alone and combined surgical-medical treatment in the management of symptomatic uterine adenomyoma. Fertil Steril 2009;92:876-85.

14. Falcone T, Goldberg JM, Margossian H, Stevens L. Robotic-assisted laparoscopic microsurgical tubal anastomosis: a human pilot study. Fertil Steril 2000;73:1040-2.

15. Sinha R, Sanjay M, Rupa B, Kumari S. Robotic surgery in gynecology. J Minim Access Surg 2015;11:50-9.
16. Lim PC, Kang E, Park DH. A comparative detail analysis of the learning curve and surgical outcome for robotic hysterectomy with lymphadenectomy versus laparoscopic hysterectomy with lymphadenectomy in treatment of endometrial cancer: a casematched controlled study of the first one hundred twenty two patients. Gynecol Oncol 2011;120:413-8.

17. Zanagnolo V, Garbi A, Achilarre MT, Minig L. Robot-assisted surgery in gynecologic cancers. J Minim Invasive Gynecol 2017;24:379-96.

18. Osada H. Uterine adenomyosis and adenomyoma: the surgical approach. Fertil Steril 2018;109:406-17.

19. Chung YJ, Kang SY, Choi MR, Cho HH, Kim JH, Kim MR. Robotassisted laparoscopic adenomyomectomy for patients who want to preserve fertility. Yonsei Med J 2016;57:1531-4.

20. Chong GO, Lee YH, Hong DG, Cho YL, Lee YS. Long-term efficacy of laparoscopic or robotic adenomyomectomy with or without medical treatment for severely symptomatic adenomyosis. Gynecol Obstet Invest 2016;81:346-52.

21. Barton SE, Gargiulo AR. Robot-assisted laparoscopic myomectomy and adenomyomectomy with a flexible CO2 laser device. J Robot Surg 2013;7:157-62.

22. Guise JM, McDonagh MS, Osterweil P, Nygren P, Chan BK, Helfand $M$. Systematic review of the incidence and consequences of uterine rupture in women with previous caesarean section. BMJ 2004;329:19-25.

23. Sizzi O, Rossetti A, Malzoni M, Minelli L, La Grotta F, Soranna L, et al. Italian multicenter study on complications of laparoscopic myomectomy. J Minim Invasive Gynecol 2007;14:453-62.

24. Dubuisson JB, Fauconnier A, Deffarges JV, Norgaard C, Kreiker G, Chapron C. Pregnancy outcome and deliveries following laparoscopic myomectomy. Hum Reprod 2000;15:869-73.

25. Dakin GF, Gagner M. Comparison of laparoscopic skills performance between standard instruments and two surgical robotic systems. Surg Endosc 2003;17:574-9. 